\title{
Power Games in MIMO Interference Systems
}

\author{
Gürdal Arslan, M. Fatih Demirkol, and Serdar Yüksel
}

\begin{abstract}
We consider a multi-link and multi-input-multioutput (MIMO) interference system in which each link wishes to minimize its own power by choosing its own signal vector subject to an information theoretic Quality-of-Service (QoS) requirement. Our setup leads to a multi-link game, referred to as a "power game", in which the feasible strategy set of an individual link depends on the strategies of the other links. We characterize the rates for which an equilibrium solution exists in a power game in terms of the equilibria of "capacity games" introduced in our earlier work [1]. We provide an example where the set of equilibrium rates is properly contained in the set of achievable rates. We provide a conservative estimate of the region of equilibrium rates using a minmax approach. We discuss the uniqueness of equilibrium as well as the convergence of best response dynamics (a.k.a. iterative water-filling) for all rates when the interference is sufficiently small and some other mild conditions are met. Finally, we extend our results to the case where the QoS requirements are softened.

Index Terms-Power control, MIMO systems, Co-channel interference, Ad-hoc networks, Game theory, Generalized Nash equilibrium.
\end{abstract}

\section{INTRODUCTION}

Multiple-Input Multiple-Output (MIMO) links use antenna arrays at both ends of a link to transmit multiple parallel streams in the same time and frequency channel [2], [3]. Signals transmitted and received by array elements at different physical locations are spatially separated by array processing algorithms. Depending on the channel scattering conditions, MIMO links can yield large gains in capacity of wireless systems.

Using antenna arrays at both ends of the links can also allow the network to accommodate multiple nearby links to transmit in the same time and frequency channel, i.e. through spatial multiplexing. In this scheme, multiple links, each with different transmitter-receiver pairs, are allowed to transmit in a given range possibly through multiple streams per link.

Such a multi-access network with MIMO links is referred to as a MIMO interference system and has been considered in previous studies including [1], [4]-[7], with the focus of finding the achievable rates of the links given their power and interference levels. Our earlier work [1] uses a multilink game framework to analyze the MIMO interference system where each link selfishly wishes to maximize its own

Research supported by NSF Grant \#ECCS-0547692.

G. Arslan is with the Department of Electrical Engineering, University of Hawaii at Manoa, 440 Holmes Hall, 2540 Dole Street, Honolulu, HI 96822, USA. E-mail: gurdal@hawaii.edu

M. F. Demirkol is with Turkcell Iletisim Hizmetleri, A.S., Istanbul, TURKEY. E-mail:demirkol@ieee.org

S. Yüksel is with the Department of Mathematics and Statistics, Queen's University, Kingston, Ontario, CANADA, K7L 3N6. E-mail: yuksel@mast.queensu.ca mutual information. In [1], the existence of Nash equilibrium associated with the multi-link game is established and sufficient conditions for the uniqueness of equilibrium are given. Decentralized algorithms are suggested as update strategies to determine the link parameters using only local information and reasonable computational burden. Since an equilibrium state does not necessarily maximize the total mutual information, a stream control approach is introduced to achieve a system-level coordination based on link negotiations. With the stream control approach, the system-wide efficiency of equilibrium is improved by imposing limits on the number of independent data streams of each link.

In this paper, we consider a MIMO interference network in which the objective of each link is to satisfy a certain Quality of Service (QoS) requirement, defined in terms of the achievable data rate of the link, with minimum possible total radiated power. In [8], an iterative method is used to determine link parameters at equilibrium for such a MIMO network. It is shown that the converged state does not necessarily yield the best network throughput. Previous work such as [9]-[11] have considered joint optimization of sets of co-channel links assuming that the base-station has an array antenna. However, the models considered in these papers involve the set of scalar power levels as the decision parameters in contrast to the model considered in this paper where the decision parameters are the covariance matrices of the transmitted signals.

We model the interactions among the links within the framework of noncooperative game theory and present a multi-link power game where each link's strategy has an effect on the strategy sets of the other links. As in [1], we follow a decentralized approach and assume availability of only local information; i.e. one link has knowledge of only its own channel and received interference conditions. We discuss the existence, uniqueness, and decentralized computation of generalized Nash equilibrium in multi-link power games.

The rest of this paper is organized as follows. Section II presents the system model. Section III introduces the power game setup. Section IV discusses the relationship between the power games and the so-called capacity games introduced in our earlier work. Section V is on obtaining a conservative estimate of the region of equilibrium rates. Section VI provides a discussion on relaxing the QoS requirements of the links. Section VII is devoted to best response dynamics as decentralized link adjustment algorithms. Section VIII concludes the paper. 


\section{A. Notation}

- := stands for "defined as".

$-\equiv$ stands for "identically equal to".

- E[.] denotes the expectation.

- $\dagger$ denotes the conjugate transpose.

- I denotes an identity matrix of an appropriate dimension.

- $|\mathbf{A}|$ denotes the determinant of a square matrix.

$-\operatorname{tr}(\mathbf{A})$ denotes the trace of a square matrix $\mathbf{A}$.

$--k$ denotes the set of indices other than $k$.

- $\mathbb{R}$ denotes the set of real numbers; $\mathbb{R}^{n}$ denotes the $n$-dimensional Euclidian vector space.

- $\mathcal{H}$ denotes the Hilbert space of $m \times m$ complex Hermitian matrices (where $m$ will be clear from the context).

- $\mathcal{H}_{+}$denotes the closed convex cone of positive semidefinite matrices in $\mathcal{H} ; \mathcal{H}_{+}^{n}$ denotes the $n$-times product $\mathcal{H}_{+} \times \cdots \times \mathcal{H}_{+}$.

- $\operatorname{diag}\left(a_{1}, a_{2}, \ldots\right)$ denotes the diagonal matrix whose diagonal entries are the scalars $a_{1}, a_{2}, \ldots$.

$-[a]^{+}=\max \{a, 0\}$ for a real $a$.

- $\mathcal{F}: \mathcal{X} \rightrightarrows \mathcal{Y}$ indicates that $\mathcal{F}$ is a correspondence mapping $\mathcal{X}$ into the set of subsets of $\mathcal{Y}$.

- $\operatorname{gr}(\mathcal{F})$ denotes the graph of a correspondence $\mathcal{F}: \mathcal{X} \rightrightarrows$ $\mathcal{Y}$, i.e., $\operatorname{gr}(\mathcal{F})=\{(\mathbf{X}, \mathbf{Y}) \in \mathcal{X} \times \mathcal{Y}: \mathbf{X} \in \mathcal{X}, \mathbf{Y} \in$ $\mathcal{F}(\mathbf{X})\}$.

\section{SySTEM MOdEL}

We consider an $L$-link communication system where each link is associated with a transmitter-receiver pair. Each transmitter and receiver are equipped with $N_{t}$ and $N_{r}$ antennas, respectively. We assume link $k$, for $k=1, \ldots, L$, transmits a complex signal vector $\mathbf{x}_{k}$ of dimension $N_{t}$. Consequently, a complex baseband signal vector of dimension $N_{r}$ denoted by $\mathbf{y}_{k}$ is received at the $k$-th receiver. The received signal vectors are related to the transmitted signal vectors by

$$
\mathbf{y}_{k}=\mathbf{H}_{k, k} \mathbf{x}_{k}+\sum_{\ell=1, \ell \neq k}^{L} \mathbf{H}_{k, \ell} \mathbf{x}_{\ell}+\mathbf{n}_{k}
$$

where

- $\mathbf{H}_{k, \ell}$ is the complex channel matrix of dimension $N_{r} \times$ $N_{t}$ for the link between the $\ell-$ th transmitter and the $k$-th receiver,

- $\mathbf{n}_{k}$ denotes the zero-mean circularly symmetric complex Gaussian noise vector at the $k$-th receiver with $E\left[\mathbf{n}_{k} \mathbf{n}_{k}^{\dagger}\right]=\mathbf{I}$.

To avoid trivialities, we make the following assumption throughout the paper.

\section{Assumption 2.1:}

$$
\mathbf{H}_{k, k} \neq 0, \text { for all } k=1, \ldots, L .
$$

We now consider a scenario in which the $k$-th link wishes to minimize its power

$$
E\left[\mathbf{x}_{k}^{\dagger} \mathbf{x}_{k}\right]
$$

by choosing the distribution of $\mathbf{x}_{k}$, independently of the other links, subject to a QoS constraint

$$
I\left(\mathbf{x}_{k} ; \mathbf{y}_{k}\right) \geq r_{k}
$$

where $I\left(\mathbf{x}_{k} ; \mathbf{y}_{k}\right)$ is the mutual information between the input and the output of the channel characterized by $\mathbf{H}_{k, k}$ and $r_{k} \in$ $[0, \infty)$ is a given constant. The $k-$ th link, not knowing the distributions of the signal vectors chosen by the other links, models the total interference $\sum_{\ell=1, \ell \neq k}^{L} \mathbf{H}_{k, \ell} \mathbf{x}_{\ell}$ at its receiver as a zero-mean circularly symmetric complex Gaussian noise vector. Under the modeling assumptions delineated above, the $k$-th link's power $E\left[\mathbf{x}_{k}^{\dagger} \mathbf{x}_{k}\right]$ is minimized by a zeromean circularly symmetric complex Gaussian distribution satisfying the QoS constraint (1); see [2]. Note that if all links make the same modeling assumptions then the links can choose their optimal distributions in a manner that is mutually consistent with their modeling assumptions. We assume that this is the case, and note from [2] that the mutual information of each link can now be written as

$$
I\left(\mathbf{x}_{k} ; \mathbf{y}_{k}\right)=\log _{2}\left|\mathbf{I}+\mathbf{R}_{k}^{-1 / 2} \mathbf{H}_{k, k} \mathbf{Q}_{k} \mathbf{H}_{k, k}^{\dagger} \mathbf{R}_{k}^{-1 / 2}\right|,
$$

$k=1, \ldots, L$, where $\mathbf{Q}_{k}:=E\left[\mathbf{x}_{k} \mathbf{x}_{k}^{\dagger}\right]$ is a Hermitian positive semi-definite matrix, and

$$
\mathbf{R}_{k}:=\mathbf{I}+\sum_{\ell=1, \ell \neq k}^{L} \mathbf{H}_{k, \ell} \mathbf{Q}_{\ell} \mathbf{H}_{k, \ell}^{\dagger}
$$

is the covariance matrix of the total interference and noise at the $k$-th receiver ${ }^{1}$. We furthermore assume that both the transmitter and receiver nodes of the $k$-th link have the knowledge of the whitened channel matrix $\mathbf{R}_{k}^{-1 / 2} \mathbf{H}_{k, k}$ once the other links choose their signal vectors. Now, from the perspective of each link, the problem amounts to choosing an appropriate covariance matrix with minimum trace satisfying its own QoS constraint in the presence of the other links that also want to minimize their own powers satisfying their own QoS constraints.

\section{A Power Game AND Generalized Nash EQUILIBRIUM}

The setup introduced in the previous section leads us to an $L$-link noncooperative game with cost functions

$$
J_{k}^{p}\left(\mathbf{Q}_{k}\right):=\operatorname{tr}\left(\mathbf{Q}_{k}\right),
$$

and feasible strategy sets

$$
\mathcal{F}_{k}^{p}\left(\mathbf{Q}_{-k}\right):=\left\{\mathbf{Q}_{k} \in \mathcal{H}_{+}: r_{k}-I\left(\mathbf{x}_{k} ; \mathbf{y}_{k}\right) \leq 0\right\},
$$

where

$-\mathbf{Q}_{-k}:=\left\{\mathbf{Q}_{1}, \ldots, \mathbf{Q}_{k-1}, \mathbf{Q}_{k+1}, \ldots, \mathbf{Q}_{L}\right\}$,

$-I\left(\mathbf{x}_{k} ; \mathbf{y}_{k}\right)$ is as in (2),

- $r_{k} \in[0, \infty)$ is a given constant,

for $k=1, \ldots, L$. We call the above game a power game and denote it by $\Gamma^{p}(\mathbf{H}, \mathbf{r})$ where $\mathbf{H}:=\left\{\mathbf{H}_{k, \ell}\right\}_{1 \leq k, \ell \leq L}$, and $\mathbf{r}:=\left\{r_{k}\right\}_{1 \leq k \leq L}$.

\footnotetext{
${ }^{1}$ In the remainder of the paper, we often suppress the dependence of the mutual information $I\left(\mathbf{x}_{k} ; \mathbf{y}_{k}\right)$ on the link covariance matrices $\mathbf{Q}_{1}, \ldots, \mathbf{Q}_{L}$
} 
A selfish link in such a strategic engagement would not be satisfied with its choice unless its cost is minimized given the choices of the other links. A steady state situation in which all link costs are mutually minimized is called a generalized Nash equilibrium ${ }^{2}$. For a more precise definition of equilibrium, let $B R_{k}^{p}$ denote $k$-th link's best response function, i.e.,

$$
B R_{k}^{p}\left(\mathbf{Q}_{-k}\right):=\operatorname{argmin}_{\mathbf{Q}_{k} \in \mathcal{F}_{k}^{p}\left(\mathbf{Q}_{-k}\right)} J_{k}^{p}\left(\mathbf{Q}_{k}\right),
$$

and let $B R^{p}:=\left\{B R_{k}^{p}\right\}_{1 \leq k \leq L}$ denote the composite best response function; see Proposition 1.1 in Appendix for the fact that, for any given $\mathbf{Q} \in \mathcal{H}_{+}^{L}, B R^{p}(\mathbf{Q})$ is nonempty and single-valued. Now, a profile of link strategies $\mathbf{Q}^{*}=$ $\left\{\mathbf{Q}_{1}^{*}, \ldots, \mathbf{Q}_{L}^{*}\right\}$ is called an equilibrium of $\Gamma^{p}(\mathbf{H}, \mathbf{r})$ if

$$
\mathbf{Q}^{*}=B R^{p}\left(\mathbf{Q}^{*}\right) \text {. }
$$

An equilibrium represents a steady-state situation in which no link has an incentive to unilaterally change its strategy. As such, equilibrium is a particularly useful notion when it is not practical to obtain and/or implement a system-wide optimal solution. For example, an equilibrium can emerge out of local optimizations performed by autonomous links in an ad-hoc wireless network without centralized coordination. Therefore, it is important to address the issue of existence and uniqueness of an equilibrium in a power game.

One approach used in [12] to establish the existence of equilibrium in single-input-single-output (SISO) power games is the framework of supermodular games which relies on the monotonicity of best response function. However, this approach is not readily applicable to the MIMO case. For instance, $B R^{p}$ is not always non-decreasing with respect to the partial order $\mathbf{Q}^{1} \leq \mathbf{Q}^{2} \Leftrightarrow \mathbf{Q}^{2}-\mathbf{Q}^{1} \in \mathcal{H}_{+}^{L}$, i.e.,

$$
\mathbf{Q}^{1} \leq \mathbf{Q}^{2} \nRightarrow B R^{p}\left(\mathbf{Q}^{1}\right) \leq B R^{p}\left(\mathbf{Q}^{2}\right) .
$$

An example is provided below.

Example 3.1: Consider a power game $\Gamma^{p}(\mathbf{H}, \mathbf{r})$ where $L=2, N_{r}=N_{t}=2, \mathbf{H}_{1,1}=\mathbf{I}, \mathbf{H}_{1,2}=\operatorname{diag}(1,0), r_{1}=$ 1. We have $B R_{1}^{p}(\mathbf{I} / 2)=\operatorname{diag}(\sqrt{3}-3 / 2, \sqrt{3}-1)$, whereas $B R_{1}^{p}(\mathbf{I})=\operatorname{diag}(0,1)$.

Another approach to establish the existence of an equilibrium in noncooperative games is based on Kakutani's wellknown fixed point theorem. Towards this end, we state a fundamental existence theorem specialized from Theorem 4.3.1 of [13] to our context.

Theorem 3.1 (Theorem 4.3.1 of [13]): Let us consider an $L$-player noncooperative game where the $k$-th player's strategy $\mathbf{Q}_{k}$ belongs to a subset $\mathcal{C}_{k}$ of a Euclidan space. Let $\mathcal{C}:=\times_{\ell} \mathcal{C}_{\ell}$ and $\mathcal{C}_{-k}:=\times_{\ell \neq k} \mathcal{C}_{\ell}$. Let $\mathcal{F}_{k}: \mathcal{C}_{-k} \rightrightarrows \mathcal{C}_{k}$ be the feasible strategy correspondence for player $k$ such that $\mathcal{F}_{k}(\cdot)=\left\{\mathbf{Q}_{k} \in \mathcal{C}_{k}: g_{k}\left(\mathbf{Q}_{k}, \cdot\right) \leq 0\right\}$ for some $g_{k}: \mathcal{C} \mapsto \mathbb{R}$. Let $J_{k}: \operatorname{gr}\left(\mathcal{F}_{k}\right) \mapsto \mathbb{R}$ be the cost function for player $k$. Assume, for all $k=1, \ldots, L$, that

\footnotetext{
${ }^{2}$ We henceforth refer to a generalized Nash equilibrium simply as an equilibrium.
}

(i) $\mathcal{C}_{k}$ is nonempty, convex and compact,

(ii) $g_{k}$ is continuous in $\mathcal{C}$,

(iii) for any fixed $\overline{\mathbf{Q}}_{-k} \in \mathcal{C}_{-k}, g_{k}\left(\cdot, \overline{\mathbf{Q}}_{-k}\right)$ is convex in $\mathcal{C}_{k}$,

(iv) for any fixed $\overline{\mathbf{Q}}_{-k} \in \mathcal{C}_{-k}$, there exists a $\overline{\mathbf{Q}}_{k} \in \mathcal{C}_{k}$ such that $g_{k}\left(\overline{\mathbf{Q}}_{k}, \overline{\mathbf{Q}}_{-k}\right)<0$,

(v) $J_{k}$ is continuous in $\operatorname{gr}\left(\mathcal{F}_{k}\right)$,

(vi) for any fixed $\overline{\mathbf{Q}}_{-k} \in \mathcal{C}_{-k}, J_{k}\left(\cdot, \overline{\mathbf{Q}}_{-k}\right)$ is convex in $\mathcal{F}_{k}\left(\overline{\mathbf{Q}}_{-k}\right)$.

Then, there exists an equilibrium.

Remark 3.1: Theorem 4.3.1 of [13] allows $\mathcal{F}_{k}$ to be arbitrary provided it is nonempty, closed, and convex valued in $\mathcal{C}_{-k}$ as well as it is both upper semi continuous and lower semi continuous in $\mathcal{C}_{-k}$. In Theorem 3.1, conditions imposed on $g_{k}$ are sufficient for $\mathcal{F}_{k}$ to satisfy such requirements. In particular, see Theorem 2.2.3 of [13] for the upper semi continuity requirement, and see Theorem 12 of [14] for the lower semi continuity requirement. Finally, if $\mathcal{F}_{k}(.) \equiv \overline{\mathcal{F}}$ for some fixed nonempty, closed, and convex subset $\overline{\mathcal{F}}$ of $\mathcal{C}_{k}$, for all $k=1, \ldots, L$, then assumptions (ii), (iii), (iv) of Theorem 3.1 are superfluous.

It turns out that Theorem 3.1 is not immediately applicable to a power game $\Gamma^{p}(\mathbf{H}, \mathbf{r})$. Note that, if we set $\mathcal{C}_{k}=\mathcal{H}_{+}$, $g_{k}=r_{k}-I\left(\mathbf{x}_{k} ; \mathbf{y}_{k}\right)$, and $J_{k}=J_{k}^{p}$, the assumptions (ii) through (vi) of Theorem 3.1 are satisfied, however, assumption (i) is not satisfied. The main difficulty stems from the fact that link feasible strategy sets $\mathcal{F}_{k}^{p}\left(\mathbf{Q}_{-k}\right)$, given in (4), are unbounded. Moreover, imposing bounds on link strategies in a way that is consistent with the assumptions of Theorem 3.1 is not straightforward. For example, it is not clear how to impose some additional power limitations $\bar{p}_{1}, \ldots, \bar{p}_{L}$ such that the modified strategy sets

$$
\left\{\mathbf{Q}_{k} \in \mathcal{H}_{+}: \operatorname{tr}\left(\mathbf{Q}_{k}\right) \leq \bar{p}_{k}, r_{k}-I\left(\mathbf{x}_{k} ; \mathbf{y}_{k}\right) \leq 0\right\}
$$

are nonempty for all $k=1, \ldots, L$. This prompts us to follow alternative routes towards establishing the existence of equilibrium in power games. We first observe a useful relationship with a capacity game.

\section{Relationship With A CAPACITy Game}

Let $\mathbf{p}:=\left\{p_{k}\right\}_{1 \leq k \leq L}$ be a collection of positive scalars. Consider an $L$-link noncooperative game with utility functions

$$
I\left(\mathbf{x}_{k} ; \mathbf{y}_{k}\right)(\mathbf{Q}),
$$

and feasible strategy sets

$$
\left\{\mathbf{Q}_{k} \in \mathcal{H}_{+}: \operatorname{tr}\left(\mathbf{Q}_{k}\right) \leq p_{k}\right\},
$$

for all $k=1, \ldots, L$, where $\mathbf{Q}=\left\{\mathbf{Q}_{1}, \ldots, \mathbf{Q}_{L}\right\}$, and $I\left(\mathbf{x}_{k} ; \mathbf{y}_{k}\right)$ is as in (2). Here, each link wishes to maximize its utility (6) by choosing a strategy $\mathbf{Q}_{k}$ from its feasible strategy set (7) We call this game a capacity game and denote it by $\Gamma^{c}(\mathbf{H}, \mathbf{p})$.

The equilibria of capacity games have been studied in [1]. In particular, the existence of an equilibrium in every capacity game $\Gamma^{c}(\mathbf{H}, \mathbf{p})$ has been established using the framework 
of concave games $[15]^{3}$. The following proposition, whose proof is revealed by a little thought, relates the equilibria of power games and capacity games.

Proposition 4.1: Fix H. Consider $\tilde{\mathbf{Q}} \in \mathcal{H}_{+}^{L}$, and let $\tilde{\mathbf{p}}:=$ $\left\{J_{k}^{p}\left(\tilde{\mathbf{Q}}_{k}\right)\right\}_{1 \leq k \leq L}, \tilde{\mathbf{r}}:=\left\{I\left(\mathbf{x}_{k} ; \mathbf{y}_{k}\right)(\tilde{\mathbf{Q}})\right\}_{1 \leq k \leq L}$. Then,

$\tilde{\mathbf{Q}}$ is an equilibrium of $\Gamma^{c}(\mathbf{H}, \tilde{\mathbf{p}})$ $\Leftrightarrow$

$\tilde{\mathbf{Q}}$ is an equilibrium of $\Gamma^{p}(\mathbf{H}, \tilde{\mathbf{r}})$.

This implies that a power game $\Gamma^{p}(\mathbf{H}, \mathbf{r})$ would possess an equilibrium if and only if the rate profile $\mathbf{r}$ can be achieved at an equilibrium of a capacity game $\Gamma^{c}(\mathbf{H}, \mathbf{p})$ for some power profile $\mathbf{p}$. This leads us to the question of how we can characterize the set of rate profiles that can be achieved at an equilibrium of $\Gamma^{c}(\mathbf{H}, \mathbf{p})$ for some $\mathbf{p}$.

For a fixed $\mathbf{H}$, define the set of equilibrium rates as

$$
\begin{aligned}
\mathcal{R}_{e}(\mathbf{H}):= & \left\{\left\{I\left(\mathbf{x}_{k} ; \mathbf{y}_{k}\right)\left(\mathbf{Q}^{*}\right)\right\}_{1 \leq k \leq L}: \mathbf{Q}^{*}\right. \text { is an } \\
& \text { equilibrium of } \left.\Gamma^{c}(\mathbf{H}, \mathbf{p}) \text { for some } \mathbf{p}\right\},
\end{aligned}
$$

and the set of achievable rates as

$$
\mathcal{R}_{a}(\mathbf{H}):=\left\{\left\{I\left(\mathbf{x}_{k} ; \mathbf{y}_{k}\right)(\mathbf{Q})\right\}_{1 \leq k \leq L}: \mathbf{Q} \in \mathcal{H}_{+}^{L}\right\} .
$$

Clearly, $\mathcal{R}_{e}(\mathbf{H}) \subset \mathcal{R}_{a}(\mathbf{H})$. Moreover, in some special cases, we have $\mathcal{R}_{e}(\mathbf{H})=\mathcal{R}_{a}(\mathbf{H})$.

Proposition 4.2: If $N_{r}=N_{t}=1$, then $\mathcal{R}_{e}(\mathbf{H})=\mathcal{R}_{a}(\mathbf{H})$.

Proof: It follows from the fact that, in the SISO case, $\mathbf{p}$ is the unique equilibrium of $\Gamma^{c}(\mathbf{H}, \mathbf{p})$.

It is possible, however, to find some $\mathbf{H}$ for which $\mathcal{R}_{e}(\mathbf{H})$ is a proper subset of $\mathcal{R}_{a}(\mathbf{H})$, i.e.,

$$
\mathcal{R}_{e}(\mathbf{H}) \subsetneq \mathcal{R}_{a}(\mathbf{H}) .
$$

Example 4.1: Consider the setup $L=2, N_{r}=N_{t}=$ $2, \mathbf{H}_{1,1}=\mathbf{H}_{2,2}=\mathbf{I}, \mathbf{H}_{1,2}=\mathbf{H}_{2,1}=\sqrt{\eta} \mathbf{I}$, for some $\eta \geq 0$. Any given rate profile $\left\{r_{k} \geq 0\right\}_{k=1}^{2}$ can be achieved by $\mathbf{Q}_{1}=\operatorname{diag}\left(2^{r_{1}}-1,0\right), \mathbf{Q}_{2}=\operatorname{diag}\left(0,2^{r_{2}}-1\right)$. Therefore, $\mathcal{R}_{a}(\mathbf{H})=[0, \infty)^{2}$.

However, if $\eta<1$, then the unique equilibrium of $\Gamma^{c}(\mathbf{H}, \mathbf{p})$ for a given $\mathbf{p}=\left\{p_{k} \geq 0\right\}_{k=1}^{2}$ is $\left\{p_{k} \mathbf{I} / 2\right\}_{k=1}^{2}$ with the corresponding rate profile $\left\{\log _{2}\left(1+\frac{p_{k} / 2}{1+\eta p_{-k} / 2}\right)\right\}_{k=1}^{2}$. This implies that, for $\eta<1$,

$$
\mathcal{R}_{e}(\mathbf{H})=\left\{\left\{r_{1}, r_{2}\right\}:\left(\sqrt{2^{r_{1}}}-1\right)\left(\sqrt{2^{r_{2}}}-1\right)<1 / \eta^{2}\right\} .
$$

Figure 1 illustrates $\mathcal{R}_{e}(\mathbf{H})$ for the case where $\eta=1 / 2$.

\footnotetext{
${ }^{3}$ The existence of an equilibrium in any capacity game $\Gamma^{c}(\mathbf{H}, \mathbf{p})$ also follows from Theorem 3.1.
}

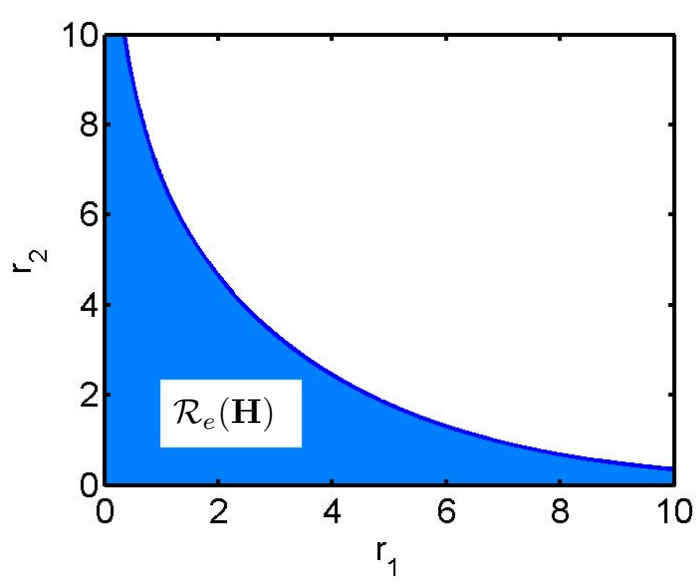

Fig. 1. An illustration of $\mathcal{R}_{e}(\mathbf{H})$ in Example 4.1 for the case $\eta=1 / 2$.

\section{A Conservative Estimate of $\mathcal{R}_{e}(\mathbf{H})$}

Here, we present an estimate of the set of equilibrium rates, which is conservative but relatively easier to compute. We recall that Theorem 3.1 is not applicable to establish an equilibrium in a power game mainly because feasible strategy sets are unbounded. To work around this issue, consider a nonempty, convex and compact subset $\mathcal{C} \in \mathcal{H}_{+}^{L}$ of the form

$$
\mathcal{C}=\mathcal{C}_{1} \times \cdots \times \mathcal{C}_{L}
$$

where, for $k=1, \ldots, L$,

$\mathcal{C}_{k}=\left\{\mathbf{Q}_{k} \in \mathcal{H}_{+}: \operatorname{tr}\left(\mathbf{Q}_{k}\right) \leq p_{k}\right.$ for some fixed $\left.p_{k}<\infty\right\}$.

Let $\mathcal{C}_{-k}:=\times_{\ell \neq k} \mathcal{C}_{\ell}$. We now define a set of minmax rates

$$
\begin{aligned}
& \mathcal{R}_{m}(\mathbf{H}):=\bigcup_{\mathcal{C}}\left\{\left\{r_{k}\right\}_{1 \leq k \leq L}: \forall 1 \leq k \leq L,\right. \\
&\left.0 \leq r_{k} \leq \min _{\mathbf{Q}_{-k} \in \mathcal{C}_{-k}} \max _{\mathbf{Q}_{k} \in \mathcal{C}_{k}} I\left(\mathbf{x}_{k} ; \mathbf{y}_{k}\right)\left(\mathbf{Q}_{k}, \mathbf{Q}_{-k}\right)\right\}
\end{aligned}
$$

where the union is taken with respect to subsets $\mathcal{C} \in \mathcal{H}_{+}^{L}$ of the form (9). Loosely speaking, $\mathcal{R}_{m}$ represents the rates that are achievable irrespective of the interference when the link strategies belong to some nonempty, convex and compact set.

\section{Proposition 5.1:}

$\mathcal{R}_{m}(\mathbf{H}) \subset \mathcal{R}_{e}(\mathbf{H}) \quad\left(\right.$ hence $\left.\mathcal{R}_{m}(\mathbf{H}) \subset \mathcal{R}_{e}(\mathbf{H}) \subset \mathcal{R}_{a}(\mathbf{H})\right)$.

Proof: For a fixed $\mathbf{H}$, consider the power game $\Gamma^{p}(\mathbf{H}, \mathbf{r})$ with $\mathbf{r} \in \mathcal{R}_{m}(\mathbf{H})$. Let $\mathcal{C}^{\mathbf{r}} \in \mathcal{H}_{+}^{L}$ be a nonempty, convex and compact subset of the form (9) such that

$$
r_{k} \leq \min _{\mathbf{Q}_{-k} \in \mathcal{C}_{-k}^{\mathbf{r}}} \max _{\mathbf{Q}_{k} \in \mathcal{C}_{k}^{\mathbf{r}}} I\left(\mathbf{x}_{k} ; \mathbf{y}_{k}\right)\left(\mathbf{Q}_{k}, \mathbf{Q}_{-k}\right), 1 \leq k \leq L .
$$

It is clear that

$$
\mathbf{Q}_{-k} \in \mathcal{C}_{-k}^{\mathbf{r}} \Rightarrow B R_{k}^{p}\left(\mathbf{Q}_{-k}\right) \in \mathcal{C}_{k}^{\mathbf{r}} .
$$

Hence, the composite best response function $B R^{p}$ maps $\mathcal{C}^{\mathbf{r}}$ into $\mathcal{C}^{\mathbf{r}}$, i.e., $B R^{p}: \mathcal{C}^{\mathbf{r}} \rightarrow \mathcal{C}^{\mathbf{r}}$. Now, by Theorem 3.1, the 
restriction of $\Gamma^{p}(\mathbf{H}, \mathbf{r})$ to $\mathcal{C}^{r}$ possesses an equilibrium, which is also an equilibrium of $\Gamma^{p}(\mathbf{H}, \mathbf{r})$. Therefore, $\mathcal{R}_{m}(\mathbf{H}) \subset$ $\mathcal{R}_{e}(\mathbf{H})$.

Proposition 5.2: Fix $\left\{\mathbf{H}_{k, k}\right\}_{1 \leq k \leq L}$ and $\mathbf{r} \in[0, \infty)^{L}$. If the interference channels are sufficiently weak, i.e., $\boldsymbol{\eta}:=$ $\left\{\mathbf{H}_{k, \ell}\right\}_{k \neq \ell}$ is sufficiently small, then

$$
\mathbf{r} \in \mathcal{R}_{m}(\mathbf{H}) \text {, and hence } \mathbf{r} \in \mathcal{R}_{e}(\mathbf{H}) \text {. }
$$

Proof: To see this, choose any $\mathbf{r}=\left\{r_{k}\right\}_{1 \leq k \leq L}$ where $r_{k} \in[0, \infty)$, for all $k=1, \ldots, L$. First consider the case where $\boldsymbol{\eta}=0$. Since $\left.I\left(\mathbf{x}_{k} ; \mathbf{y}_{k}\right)(\mathbf{Q})\right|_{\boldsymbol{\eta}=0}$ depends only on $\mathbf{Q}_{k}$, there is a strategy profile $\overline{\mathbf{Q}} \in \mathcal{H}_{+}^{L}$ such that

$$
\left.I\left(\mathbf{x}_{k} ; \mathbf{y}_{k}\right)(\overline{\mathbf{Q}})\right|_{\boldsymbol{\eta}=0}=2 r_{k} .
$$

Consider the nonempty, convex and compact subset $\mathcal{C}^{2 \mathbf{r}}:=$ $\mathcal{C}_{1}^{2 \mathbf{r}} \times \cdots \times \mathcal{C}_{L}^{2 \mathbf{r}}$ where

$$
\mathcal{C}_{k}^{2 \mathbf{r}}=\left\{\mathbf{Q}_{k} \in \mathcal{H}_{+}: \operatorname{tr}\left(\mathbf{Q}_{k}\right) \leq \operatorname{tr}\left(\overline{\mathbf{Q}}_{k}\right)\right\}, k=1, \ldots, L .
$$

Since $I\left(\mathbf{x}_{k} ; \mathbf{y}_{k}\right)(\mathbf{Q})$ is continuous with respect to $\boldsymbol{\eta}$ at $\boldsymbol{\eta}=0$, for sufficiently small $\boldsymbol{\eta}$,

$$
r_{k} \leq \min _{\mathbf{Q}_{-k} \in \mathcal{C}_{-k}^{2 \mathbf{r}}} \max _{\mathbf{Q}_{k} \in \mathcal{C}_{k}^{2 \mathbf{r}}} I\left(\mathbf{x}_{k} ; \mathbf{y}_{k}\right)\left(\mathbf{Q}_{k}, \mathbf{Q}_{-k}\right), 1 \leq k \leq L
$$

The following proposition, whose proof follows from the definition of $\mathcal{R}_{m}(\mathbf{H})$ and Proposition 5.1, states that all sufficiently small rates are equilibrium rates.

Proposition 5.3: Fix H. There exist $\bar{r}_{k}>0, k=1, \ldots, L$, such that

$$
\left\{\left\{r_{k}\right\}_{1 \leq k \leq L}: r_{k} \in\left[0, \bar{r}_{k}\right], k=1, \ldots, L\right\} \subset \mathcal{R}_{e}(\mathbf{H}) .
$$

It turns out that, for some $\mathbf{H}, \mathcal{R}_{m}(\mathbf{H})$ is a proper subset of $\mathcal{R}_{e}(\mathbf{H})$, i.e.,

$$
\mathcal{R}_{m}(\mathbf{H}) \subsetneq \mathcal{R}_{e}(\mathbf{H})
$$

Example 5.1: Consider the setup in Example 4.1. Simple calculations yield, for all $\eta \geq 0$,

$$
\mathcal{R}_{m}(\mathbf{H})=\left\{\left\{r_{1}, r_{2}\right\}:\left(\sqrt{2^{r_{1}}}-1\right)\left(\sqrt{2^{r_{2}}}-1\right)<1 / \eta^{2}\right\} .
$$

In view of Example 4.1, this means that, for $\eta<1$, $\mathcal{R}_{m}(\mathbf{H})=\mathcal{R}_{e}(\mathbf{H})$.

However, for $\eta \geq 1$, the capacity game $\Gamma^{c}(\mathbf{H}, \mathbf{p})$ for some fixed $\mathbf{p}=\left\{p_{k}>0\right\}_{k=1}^{2}$ has additional equilibria (in addition to $\left\{p_{1} \mathbf{I} / 2, p_{2} \mathbf{I} / 2\right\}$ ). In particular, when $\eta \geq 1$ and $p_{1}=p_{2}=p$ for some $p>0,\{\operatorname{diag}(p, 0), \operatorname{diag}(0, p)\}$ is such an additional equilibrium with the rate profile $\left\{\log _{2}(1+p), \log _{2}(1+p)\right\}$. For a sufficiently large $p>0$, $\left\{\log _{2}(1+p), \log _{2}(1+p)\right\} \notin \mathcal{R}_{m}(\mathbf{H})$. As a result, for $\eta \geq 1$, $\mathcal{R}_{m}(\mathbf{H}) \subsetneq \mathcal{R}_{e}(\mathbf{H})$.

\section{SOFTEnING QoS REQUIREMENTS}

We thus far viewed the QoS requirements as hard constraints. One of the difficulties with this viewpoint is that if the rates that the links seek to achieve are not achievable at an equilibrium, then the links would not be able to settle at any solution. In a practical scenario, an individual link not knowing the entire setup would not be able to easily determine whether or not its QoS requirement is achievable at an equilibrium. The lack of an equilibrium would manifest itself as persistent oscillations when the links continually adjust their covariance matrices using an update algorithm such as best response dynamics. To overcome this difficulty, we now soften the QoS requirements by removing the hard constraint $\mathbf{Q}_{k} \in \mathcal{F}_{k}^{p}\left(\mathbf{Q}_{-k}\right)$ and modifying the link cost functions as

$$
J_{k}^{w}(\mathbf{Q}):=\operatorname{tr}\left(\mathbf{Q}_{k}\right)+w\left[r_{k}-I\left(\mathbf{x}_{k} ; \mathbf{y}_{k}\right)\right]^{+}, \quad \mathbf{Q}_{k} \in \mathcal{H}_{+},
$$

where $w \in[0, \infty)$ is the cost of violating the QoS requirements. We refer to a game characterized by the cost functions $J_{1}^{w}, \ldots, J_{L}^{w}$ and the feasible strategy sets $\mathcal{H}_{+} \times \cdots \times \mathcal{H}_{+}$as a "weighted power game" and denote it by $\Gamma^{w}(\mathbf{H}, \mathbf{r})$.

We denote the $k$-th link's best response function by $B R_{k}^{w}$, i.e.,

$$
B R_{k}^{w}\left(\mathbf{Q}_{-k}\right)=\operatorname{argmin}_{\mathbf{Q}_{k} \in \mathcal{H}_{+}} J_{k}^{w}\left(\mathbf{Q}_{k}, \mathbf{Q}_{-k}\right),
$$

and the composite best response function by $B R^{w}:=$ $\left\{B R_{k}^{w}\right\}_{1 \leq k \leq L}$; see Proposition 1.1 in Appendix for the fact that, for any given $\mathbf{Q} \in \mathcal{H}_{+}^{L}, B R^{w}(\mathbf{Q})$ is nonempty and single-valued. We call a profile of link strategies $\check{\mathbf{Q}} \in \mathcal{H}_{+}^{L}$ an equilibrium of $\Gamma^{w}(\mathbf{H}, \mathbf{r})$ if

$$
\check{\mathbf{Q}}=B R^{w}(\check{\mathbf{Q}}) \text {. }
$$

We are essentially interested in the case where $w \uparrow \infty$, since, for large $w$, the links are expected to strive towards achieving their QoS requirements. If it is not possible for a link to achieve its QoS requirement, then this would result in a very high power level for the particular link at an equilibrium, which would perhaps prompt the link to scale down its QoS requirement. One advantage of softening the QoS requirements is that an equilibrium would always exist regardless of the rates that the links seek to achieve. Another advantage is that if the target rates are equilibrium rates, then the equilibria of a weighted power game for sufficiently large $w$ contain the equilibria of the corresponding power game which by definition satisfy the QoS requirements.

Proposition 6.1: Fix $\mathbf{H}, \mathbf{r} \in[0, \infty)^{L}$, and $w \in[0, \infty)$. Then, the following statements are true.

1) The weighted power game $\Gamma^{w}(\mathbf{H}, \mathbf{r})$ possesses an equilibrium.

2) An equilibrium of $\Gamma^{w}(\mathbf{H}, \mathbf{r})$ satisfying the QoS requirement (1) is also an equilibrium of $\Gamma^{p}(\mathbf{H}, \mathbf{r})$.

3) There exists a $\bar{w} \in[0, \infty)$ such that if $w \in[\bar{w}, \infty)$ then any equilibrium of $\Gamma^{p}(\mathbf{H}, \mathbf{r})$ is also an equilibrium of $\Gamma^{w}(\mathbf{H}, \mathbf{r})$.

4) Let $\left\{w_{n}\right\}_{n \geq 1}$ be an increasing positive-valued and unbounded sequence of scalars. Let $\left\{\mathbf{Q}^{n}\right\}_{n \geq 1}$ be a 
corresponding sequence in $\mathcal{H}_{+}^{L}$ such that, for all $n \geq 1$, $\mathbf{Q}^{n}$ is an equilibrium of $\Gamma^{w_{n}}(\mathbf{H}, \mathbf{r})$.

a) If $\sup _{n \geq 1} \sum_{k=1}^{L} \operatorname{tr}\left(\mathbf{Q}_{k}^{n}\right)<\infty$, then there exists an $\bar{n} \geq 1$ such that, for all $n \geq \bar{n}, \mathbf{Q}^{n}$ is an equilibrium of $\Gamma^{p}(\mathbf{H}, \mathbf{r})$.

b) If $\mathbf{r} \notin \mathcal{R}_{e}(\mathbf{H})$, then $\sup _{n \geq 1} \sum_{k=1}^{L} \operatorname{tr}\left(\mathbf{Q}_{k}^{n}\right)=\infty$.

Proof:

1) We observe that, for any $\mathbf{Q}_{-k} \in \mathcal{H}_{+}^{L-1}$,

$$
\begin{aligned}
\operatorname{tr}\left(B R_{k}^{w}\left(\mathbf{Q}_{k}\right)\right) & \leq J_{k}^{w}\left(B R_{k}^{w}\left(\mathbf{Q}_{k}\right), \mathbf{Q}_{-k}\right) \\
& \leq J_{k}^{w}\left(0, \mathbf{Q}_{-k}\right)=w r_{k} .
\end{aligned}
$$

Therefore, without loss of generality, we can restrict the link strategies to

$$
\mathcal{F}^{w}:=\mathcal{F}_{1}^{w} \times \cdots \times \mathcal{F}_{L}^{w},
$$

where, for $k=1, \ldots, L$,

$$
\mathcal{F}_{k}^{w}:=\left\{\mathbf{Q}_{k} \in \mathcal{H}_{+}: \operatorname{tr}\left(\mathbf{Q}_{k}\right) \leq w r_{k}\right\} .
$$

Clearly, $\mathcal{F}_{k}^{w}$ is nonempty, convex and compact. Also, $J_{k}^{w}$ is continuous in $\mathcal{F}^{w}$, and $J_{k}^{w}\left(\cdot, \mathbf{Q}_{-k}\right)$ is convex in $\mathcal{F}_{k}^{w}$ for each fixed $\mathbf{Q}_{-k} \in \mathcal{F}_{-k}^{w}:=X_{\ell \neq k} \mathcal{F}_{\ell}^{w}$. Now, the existence of an equilibrium follows from Theorem 3.1.

2) Obvious.

3) Consider any equilibrium $\left\{\mathbf{Q}_{k}^{*}\right\}_{k=1}^{L}$ of the power game $\Gamma^{p}(\mathbf{H}, \mathbf{r})$. Proposition 1.1 in Appendix shows that there exists a $\bar{w} \in[0, \infty)$ such that, for all $w \in[\bar{w}, \infty)$,

$$
B R_{k}^{w}\left(\mathbf{Q}_{-k}^{*}\right)=B R_{k}^{p}\left(\mathbf{Q}_{-k}^{*}\right)=\mathbf{Q}_{k}^{*}
$$

for all $k=1, \ldots, L$.

4) a) Since $\left\{\mathbf{Q}^{n}\right\}_{n \geq 1}$ belongs to a compact subset of $\mathcal{H}_{+}^{L}$, there exists a $\bar{w} \geq 0$ such that, for all $w \geq \bar{w}, B R_{k}^{w}\left(\mathbf{Q}_{-k}^{n}\right)=B R_{k}^{p}\left(\mathbf{Q}_{-k}^{n}\right), n \geq 1, k=$ $1, \ldots, L$; see Proposition 1.1 in Appendix . This implies that there exists an $\bar{n} \geq 1$, such that, for all $n \geq \bar{n}, \mathbf{Q}_{k}^{n}=B R_{k}^{w_{n}}\left(\mathbf{Q}_{-k}^{n}\right)=B R_{k}^{p}\left(\mathbf{Q}_{-k}^{n}\right)$, $k=1, \ldots, L$.

b) Suppose that $\sup _{n \geq 1} \sum_{k=1}^{L} \operatorname{tr}\left(\mathbf{Q}_{k}\right)<\infty$. Then, part a) implies that, for a sufficiently large $n, \mathbf{Q}^{n}$ is an equilibrium of $\Gamma^{P}(\mathbf{H}, \mathbf{r})$, which contradicts $\mathbf{r} \notin \mathcal{R}_{e}(\mathbf{H})$.

\section{BEST RESPONSE DYNAMICS}

Here, we consider a situation in which the links are iteratively adjusting their covariance matrices to minimize their cost functions in a weighted power game. During iteration $t+1$, any individual link $k$ knows nothing about the setup except it can compute its own best response $B R_{k}^{w}\left(\mathbf{Q}_{-k}(t)\right)$ to the decisions $\mathbf{Q}_{-k}(t)$ made by the other links at the previous iteration $t$. For this, it is sufficient for an individual link $k$ to know its own channel matrix $\mathbf{H}_{k, k}$, its own QoS requirement $r_{k}$, and to measure the covariance matrix $\mathbf{R}_{k}$ of the total noise and interference corresponding to $\mathbf{Q}_{-k}(t)$. The actual computation of $B R_{k}^{w}\left(\mathbf{Q}_{-k}(t)\right)$ can be done as shown in Proposition 1.1 in Appendix . Once $B R_{k}^{w}\left(\mathbf{Q}_{-k}(t)\right)$ is obtained, any link $k$ updates its own covariance matrix according to

$$
\mathbf{Q}_{k}(t+1)=\left(1-\alpha_{k}(t)\right) \mathbf{Q}_{k}(t)+\alpha_{k}(t) B R_{k}^{w}\left(\mathbf{Q}_{-k}(t)\right),
$$

where $0 \leq \alpha_{k}(t) \leq 1$ is a parameter that represents the $k$-th link's willingness to optimize (in other words, $1-\alpha_{k}(t)$ is the $k$-th link's inertia) at step $t$. The inertia prevents the links from overreacting and generally helps with the convergence of the updates.

We also consider the case where the links are engaged in a power game and therefore update their covariance matrices according to

$$
\mathbf{Q}_{k}(t+1)=\left(1-\alpha_{k}(t)\right) \mathbf{Q}_{k}(t)+\alpha_{k}(t) B R_{k}^{p}\left(\mathbf{Q}_{-k}(t)\right),
$$

where $0 \leq \alpha_{k}(t) \leq 1$ again represents the $k$-th link's willingness to optimize at step $t$; see Proposition 1.1 in Appendix for the actual computation of $B R_{k}^{p}\left(\mathbf{Q}_{-k}(t)\right)$. We should point out that the covariance matrices generated during the iterations of (13) need not satisfy the QoS requirements of the links. More precisely, $\mathbf{Q}_{k}(t)$ need not belong to $\mathcal{F}_{k}^{p}\left(\mathbf{Q}_{-k}(t)\right)$ even though $\mathbf{Q}_{k}(t) \in \mathcal{F}_{k}^{p}\left(\mathbf{Q}_{-k}(t-1)\right)$ by construction. However, if best response dynamics (13) converge to some limiting covariance matrices $\tilde{\mathbf{Q}}$, then $\tilde{\mathbf{Q}}$ must be an equilibrium (and satisfy all QoS requirements).

Best response dynamics (12)-(13) can be generalized by allowing the links to update intermittently as long as they do not completely stop updating until convergence. In our numerical simulations, both best response dynamics (12)(13) typically converge. Moreover, we believe that, for sufficiently small interference, convergence can be proven along the lines of Proposition 4.1 in [1] by showing that best response functions are contractions.

Conjecture 7.1: Fix $\left\{\mathbf{H}_{k, k}\right\}_{1 \leq k \leq L}, \mathbf{r} \in[0, \infty)^{L}$, and $w \in$ $[0, \infty)$. Assume

(i) $\operatorname{rank}\left(\mathbf{H}_{k, k}\right)=N_{t}$, for all $k=1, \ldots, L$,

(ii) $\lim _{t \rightarrow \infty} \alpha_{k}(t)=0$ and $\sum_{k=0}^{\infty} \alpha_{k}(t)=\infty$, for all $k=$ $1, \ldots, L$.

If the interference channels are sufficiently weak, i.e., $\left\{\mathbf{H}_{k, \ell}\right\}_{k \neq \ell}$ is sufficiently small, then the following statements are true.

1) Best response dynamics (12) globally converge to the unique equilibrium of the weighted power game $\Gamma^{w}(\mathbf{H}, \mathbf{r})$.

2) Best response dynamics (13) locally converge to the unique equilibrium of the power game $\Gamma^{p}(\mathbf{H}, \mathbf{r})$.

Remark 7.1: Note that, in addition to convergence, Conjecture 7.1 states the existence of a unique equilibrium in weighted power games and power games under certain conditions.

We now provide some examples of nonconvergent cases when the conditions of Proposition 7.1 are not met. 
Example 7.1: Best-response dynamics with $\alpha_{k}(t)=1$ starting from 0 may diverge even if an equilibrium exists. Consider the setup $L=2, N_{r}=N_{t}=2, \mathbf{H}_{1,1}=\mathbf{H}_{2,2}=$ $\mathbf{I}, \mathbf{H}_{1,2}=\mathbf{H}_{2,1}=\sqrt{\eta} \mathbf{I}$, for some $\eta \geq 0$, and $r_{1}=r_{2}=1$. We fist compute that, for any $\rho \geq 0, B R_{k}^{p}(\rho \mathbf{I})=\gamma(1+\rho \eta) \mathbf{I}$, where $\gamma:=\sqrt{2}-1$. Therefore, starting from 0 , best-response dynamics would generate

$$
\begin{aligned}
0 \rightarrow \gamma\{\mathbf{I}, \mathbf{I}\} \rightarrow \gamma(1 & +\gamma \eta)\{\mathbf{I}, \mathbf{I}\} \\
& \rightarrow \gamma\left(1+\gamma \eta+\gamma^{2} \eta^{2}\right)\{\mathbf{I}, \mathbf{I}\} \rightarrow \ldots
\end{aligned}
$$

which diverges when $\gamma \eta \geq 1$.

Example 7.2: Best-response dynamics may not converge even if the initial condition is feasible and arbitrarily close to an equilibrium. Consider the setup given in the previous example with $\eta=1$, in which $(\operatorname{diag}(1,0), \operatorname{diag}(0,1))$ is an equilibrium. We first compute that, for any $\theta \geq 0$ and small $\epsilon>0$

$$
\begin{aligned}
& B R_{k}^{p}(\operatorname{diag}(\theta, \theta+1-\epsilon))=\operatorname{diag}(\bar{\theta}+1-\epsilon, \bar{\theta}) \\
& B R_{k}^{p}(\operatorname{diag}(\theta+1-\epsilon, \theta))=\operatorname{diag}(\bar{\theta}, \bar{\theta}+1-\epsilon),
\end{aligned}
$$

for some $\bar{\theta}>0$. Now, consider the initial condition $\mathbf{Q}^{0}=$ $(\operatorname{diag}(1, \epsilon), \operatorname{diag}(0,1+\epsilon))$ for some small $\epsilon>0$. Note that $\mathbf{Q}^{0}$ is feasible, i.e., $I\left(\mathbf{x}_{k} ; \mathbf{y}_{k}\right)\left(\mathbf{Q}^{0}\right) \geq 1$. Starting from $\mathbf{Q}^{0}$, best-response dynamics would generate

$$
\begin{aligned}
\mathbf{Q}^{1} & =\left\{\operatorname{diag}(1,0), \operatorname{diag}\left(\theta^{1}, \theta^{1}+1-\epsilon\right)\right\} \\
\mathbf{Q}^{2} & =\left\{\operatorname{diag}\left(\theta^{2}+1-\epsilon, \theta^{2}\right), \operatorname{diag}(0,1)\right\} \\
\mathbf{Q}^{3} & =\left\{\operatorname{diag}(1,0), \operatorname{diag}\left(\theta^{3}, \theta^{3}+1-\epsilon\right)\right\} \\
& \vdots
\end{aligned}
$$

for some $\theta^{1}>0, \theta^{2}>0, \theta^{3}>0, \ldots$

\section{CONCLUSIONS}

We studied a power control problem in MIMO interference systems within the framework of multi-link games. We established a relationship between power control and capacity control problems from a game theoretic perspective. We illustrated on an example that equilibrium may not exist for all achievable rates. Using a minmax approach, we obtained a conservative estimate of the rate region for which an equilibrium exists in a power game. We discussed some sufficient conditions for the uniqueness of equilibrium as well as for the convergence of best-response process (iterative water-filling). We extended our results to the case where QoS requirements are softened. Improving the efficiency of equilibrium using stream control as in [1] remains as a significant future work.

\section{REFERENCES}

[1] G. Arslan, M. F. Demirkol, and Y. Song, "Equilibrium efficiency improvement in MIMO interference systems: a decentralized stream control approach," IEEE Transactions on Wireless Communications, vol. 6, no. 8, pp. 2984-2993, 2007.

[2] E. Telatar, "Capacity of multi-antenna gaussian channels," European Transactions on Telecommunications, vol. 10, no. 6, pp. 585-595, 1999.
[3] G. J. Foschini and M. J. Gans, "On limits of wireless communications in a fading environment when using multiple antennas," Wireless Personal Communications, vol. 6, pp. 311-335, 1998.

[4] M. F. Demirkol and M. A. Ingram, "Power-controlled capacity for interfering MIMO links," in Proceedings of the IEEE Vehicular Technology Conference, vol. 1, Oct. 2001, pp. 187-191.

[5] — "Stream control in networks with interfering MIMO links," in Proceedings of the IEEE Wireless Communications and Networking Conference, vol. 1, Mar. 2003, pp. 343-348.

[6] S. Ye and R. S. Blum, "Optimized signalling for MIMO interference systems with feedback," IEEE Transactions on Signal Processing, vol. 51, no. 11, pp. 2839-2848, 2003.

[7] G. Scutari, D. P. Palomar, and S. Barbarossa, "Competitive design of multiuser mimo systems based on game theory: a unified view," Journal of Selected Areas in Communications, vol. 26, no. 7, pp. 10891103, Sept. 2008.

[8] M. F. Demirkol and M. A. Ingram, "Control using capacity constraints for interfering MIMO links," in Proceedings of the International Symposium on Personal, Indoor and Mobile Radio Communications, vol. 3, Sept. 2001, pp. 1032-1036.

[9] J.-S. Pang, G. Scutari, F. Facchinei, and C. Wang, "Distributed power allocation with rate constraints in Gaussian parallel interference channels," IEEE Transaction on Information Theory, vol. 54, no. 8, pp. 3471-3489, Aug. 2008.

[10] F. R. Farrokhi, K. J. R. Liu, and L. Tassiulas, "Transmit beamforming and power control for cellular wireless systems," Journal of Selected Areas in Communications, vol. 16, no. 8, pp. 1437-1450, Oct. 1998.

[11] G. J. Foschini and Z. Miljanic, "A simple distributed autonomous power control algorithm and its convergence," IEEE Transactions on Vehicular Technology, vol. 42, no. 4, pp. 641-646, Nov. 1993.

[12] E. Altman and Z. Altman, "S-modular games and power control in wireless networks," IEEE Transactions on Automatic Control, vol. 48 , no. 5, pp. 839-842, 2003.

[13] T. Ichiishi, Game Theory for Economic Analysis. New York, NY: Academic Press, 1983.

[14] W. W. Hogan, "Point-to-set mappings in mathematical programming," SIAM Review, vol. 15, no. 13, pp. 591-603, July 1973.

[15] J. B. Rosen, "Existence and uniqueness of equilibrium points for concave $n$-person games," Econometrica, vol. 33, no. 3, pp. 520 534, 1965.

\section{APPENDIX I \\ BEST RESPONSE FUNCTIONS}

Proposition 1.1: Fix $\mathbf{H}, \mathbf{r} \in[0, \infty)^{L}$, and $w \in[0, \infty)$.

1) The $k$-th link's best response in $\Gamma^{w}(\mathbf{H}, \mathbf{r})$ to any fixed $\mathbf{Q}_{-k} \in \mathcal{H}_{+}$is unique and given as

$$
\begin{aligned}
B R_{k}^{w} & \left(\mathbf{Q}_{-k}\right) \\
& =\mathbf{V}_{k} \operatorname{diag}\left(q_{k}^{1,1}, \ldots, q_{k}^{n_{k}, n_{k}}, 0, \ldots, 0\right) \mathbf{V}_{k}^{\dagger} \\
& =\sum_{i=1}^{n_{k}} q_{k}^{i, i} \mathbf{v}_{k}^{i}\left(\mathbf{v}_{k}^{i}\right)^{\dagger}
\end{aligned}
$$

where

$$
\text { - } \sigma_{k}^{1} \geq \cdots \geq \sigma_{k}^{n_{k}}>\sigma_{k}^{n_{k}+1}=\cdots=\sigma_{k}^{N_{t}}=0
$$
are eigenvalues of $\mathbf{H}_{k, k}^{\dagger} \mathbf{R}_{k}^{-1} \mathbf{H}_{k, k}\left(\sigma_{k}^{1}>0\right.$ due to Assumption 2.1, and $1 \leq n_{k} \leq \min \left\{N_{r}, N_{t}\right\}$ ),

- $\mathbf{V}_{k}$ is a unitary matrix such that its columns $\mathbf{v}_{k}^{1}, \ldots, \mathbf{v}_{k}^{N_{t}}$ are a set of orthonormal eigenvectors of $\mathbf{H}_{k, k}^{\dagger} \mathbf{R}_{k}^{-1} \mathbf{H}_{k, k}$ corresponding to $\sigma_{k}^{1}, \ldots, \sigma_{k}^{N_{t}}$, respectively,

- $q_{k}^{i, i}=\left[\min \left\{w / \ln 2, \mu_{k}\right\}-1 / \sigma_{k}^{i}\right]^{+}$, for all $i=$ $1, \ldots, n_{k}$,

- $\mu_{k}$ is such that $r_{k}=\sum_{i=1}^{n_{k}}\left[\log _{2}\left(\mu_{k} \sigma_{k}^{i}\right)\right]^{+}$. 
2) The $k$-th link's best response in $\Gamma^{p}(\mathbf{H}, \mathbf{r})$ to any fixed $\mathbf{Q}_{-k} \in \mathcal{H}_{+}$is unique and given as

$$
\begin{aligned}
B R_{k}^{p} & \left(\mathbf{Q}_{-k}\right) \\
& =\mathbf{V}_{k} \operatorname{diag}\left(\tilde{q}_{k}^{1,1}, \ldots, \tilde{q}_{k}^{n_{k}, n_{k}}, 0, \ldots, 0\right) \mathbf{V}_{k}^{\dagger} \\
& =\sum_{i=1}^{n_{k}} \tilde{q}_{k}^{i, i} \mathbf{v}_{k}^{i}\left(\mathbf{v}_{k}^{i}\right)^{\dagger},
\end{aligned}
$$

where $\tilde{q}_{k}^{i, i}=\left[\mu_{k}-1 / \sigma_{k}^{i}\right]^{+}, i=1, \ldots, n_{k}$, and $\sigma_{k}^{1}, \ldots, \sigma_{k}^{N_{t}}, \mathbf{V}_{k}=\left[\mathbf{v}_{k}^{1}, \ldots, \mathbf{v}_{k}^{N_{t}}\right], \mu_{k}$ are as in the first part. Note that $B R_{k}^{p}\left(\mathbf{Q}_{-k}\right)=B R_{k}^{w}\left(\mathbf{Q}_{-k}\right)$ whenever $w / \ln 2 \geq \mu_{k}$.

Proof:

To avoid trivialities, we assume $w>0$ and $r_{k}>0$.

1) Let $\boldsymbol{\Sigma}:=\operatorname{diag}\left(\sigma_{k}^{1}, \ldots, \sigma_{k}^{n_{k}}, 0, \ldots, 0\right)$. Using the determinant identity $|\mathbf{I}+\mathbf{A B}|=|\mathbf{I}+\mathbf{B A}|$, we write

$$
\begin{aligned}
& J_{k}^{w}\left(\mathbf{Q}_{k}, \mathbf{Q}_{-k}\right)=\operatorname{tr}\left(\mathbf{V}_{k}^{\dagger} \mathbf{Q}_{k} \mathbf{V}_{k}\right) \\
& \quad+w\left[r_{k}-\log _{2}\left|\mathbf{I}+\boldsymbol{\Sigma}_{k}^{1 / 2} \mathbf{V}_{k}^{\dagger} \mathbf{Q}_{k} \mathbf{V}_{k} \boldsymbol{\Sigma}_{k}^{1 / 2}\right|\right]^{+} .
\end{aligned}
$$

We can equivalently perform the minimization over $\overline{\mathbf{Q}}_{k}:=\mathbf{V}_{k}^{\dagger} \mathbf{Q}_{k} \mathbf{V}_{k}$, i.e.,

$$
\begin{aligned}
& B R_{k}^{w}\left(\mathbf{Q}_{-k}\right)=\left\{\mathbf{V}_{k} \overline{\mathbf{Q}}_{k} \mathbf{V}_{k}^{\dagger}:\right. \\
& \overline{\mathbf{Q}}_{k} \in \operatorname{argmin}_{\mathbf{Q}_{k} \in \mathcal{H}_{+}}\left\{\operatorname{tr}\left(\mathbf{Q}_{k}\right)+\right. \\
& \left.\left.w\left[r_{k}-\log _{2}\left|\mathbf{I}+\boldsymbol{\Sigma}_{k}^{1 / 2} \mathbf{Q}_{k} \boldsymbol{\Sigma}_{k}^{1 / 2}\right|\right]^{+}\right\}\right\} .
\end{aligned}
$$

Let $q_{k}^{1,1}, \ldots, q_{k}^{N_{t}, N_{t}}$ be the diagonal entries of $\mathbf{Q}_{k}$. By Hadamard's inequality for Hermitian positive definite matrices, $\left|\mathbf{I}+\boldsymbol{\Sigma}_{k}^{1 / 2} \mathbf{Q}_{k} \boldsymbol{\Sigma}_{k}^{1 / 2}\right| \leq \prod_{i=1}^{n_{k}}\left(1+q_{k}^{i, i} \sigma_{k}^{i}\right)$ with equality if and only if $\mathbf{I}+\boldsymbol{\Sigma}_{k}^{1 / 2} \mathbf{Q}_{k} \boldsymbol{\Sigma}_{k}^{1 / 2}$ is diagonal. This implies that the minimum above can be achieved only by diagonal matrices in $\mathcal{H}_{+}$of the form $\operatorname{diag}\left(q_{k}^{1,1}, \ldots, q_{k}^{n_{k}, n_{k}}, 0, \ldots, 0\right)$. Accordingly, we focus on

$$
\begin{aligned}
\min _{q_{k}^{1,1} \geq 0, \ldots, q_{k}^{n_{k}, n_{k}} \geq 0} & \left\{\sum_{i=1}^{n_{k}} q_{k}^{i, i}\right. \\
+w & {\left.\left[r_{k}-\sum_{i=1}^{n_{k}} \log _{2}\left(1+q_{k}^{i, i} \sigma_{k}^{i}\right)\right]^{+}\right\} . }
\end{aligned}
$$

Ignoring [. $]^{+}$in (14) leads to a strictly convex cost function over the positive orthant with the unique minimizer

$$
\check{q}_{k}^{i, i}=\left[w / \ln 2-1 / \sigma_{k}^{i}\right]^{+}, i=1, \ldots, n_{k} .
$$

Since ignoring [.] ${ }^{+}$in (14) lower bounds the cost, if $r_{k} \geq \sum_{i=1}^{n_{k}} \log _{2}\left(1+\tilde{q}_{k}^{i, i} \sigma_{k}^{i}\right)$, then the unique minimizer of (14) is given by (15). On the other hand, if $r_{k}<$ $\sum_{i=1}^{n_{k}} \log _{2}\left(1+\tilde{q}_{k}^{i, i} \sigma_{k}^{i}\right)$, then the unique minimizer of (14) is given as

$$
\hat{q}_{k}^{i, i}=\left[\mu_{k}-1 / \sigma_{k}^{i}\right]^{+}, i=1, \ldots, n_{k},
$$

where $\mu_{k}$ is such that $r_{k}=\sum_{i=1}^{n_{k}} \log _{2}\left(1+\hat{q}_{k}^{i, i} \sigma_{k}^{i}\right) \Leftrightarrow$ $r_{k}=\sum_{i=1}^{n_{k}}\left[\log _{2}\left(\mu_{k} \sigma_{k}^{i}\right)\right]^{+}$. Finally, for the uniqueness of $B R_{k}^{p}\left(\mathbf{Q}_{-k}\right)$, note that, even though the choice of the eigenvectors $\mathbf{v}_{k}^{1}, \ldots, \mathbf{v}_{k}^{n_{k}}$ is not unique, for an $m$-repeated eigenvalue, say $\sigma_{k}^{1}=\cdots=\sigma_{k}^{m}>0$, the matrix $\sum_{i=1}^{m} \mathbf{v}_{k}^{i}\left(\mathbf{v}_{k}^{i}\right)^{\dagger}$ is unique, i.e., independent of the choice of the eigenvectors $\mathbf{v}_{k}^{1}, \ldots, \mathbf{v}_{k}^{m}$.

2) Obvious from the first part. 